\title{
Imaging Quality Assurance Review Center
}

National Cancer Institute

\section{Source}

National Cancer Institute. Imaging Quality Assurance Review Center. NCI Thesaurus.

Code C103911.

A research program that provides specialized radiotherapy quality assurance and diagnostic imaging data management services. 